Published in final edited form as:

J Crit Care. 2018 April ; 44: 18-23. doi:10.1016/j.jcrc.2017.10.003.

\title{
Single intervention for a reduction in portable chest radiography (pCXR) in cardiovascular and surgical/trauma ICUs and associated outcomes $\star$
}

\author{
Joseph E. Tonna ${ }^{a, b,{ }^{*}, 1}$, Kensaku Kawamoto $^{c, 2}$, Angela P. Presson ${ }^{\mathrm{d}, 2}$, Chong Zhang ${ }^{\mathrm{d}, 2}$, Mary \\ C. Mone ${ }^{\mathrm{g}, 2}$, Robert E. Glasgow ${ }^{\mathrm{g}, 3}$, Richard G. Bartong ${ }^{\mathrm{g}, 3}$, John R. Hoidal ${ }^{\mathrm{e}, 2}$, and Yoshimi \\ Anzai ${ }^{\mathrm{f}, 2}$ \\ aDivision of Cardiothoracic Surgery, Department of Surgery, University of Utah, Salt Lake City, UT, \\ United States \\ bDivision of Emergency Medicine, Department of Surgery, University of Utah, Salt Lake City, UT, \\ United States \\ 'Department of Biomedical Informatics, University of Utah, Salt Lake City, UT, United States \\ ${ }^{\mathrm{d} D i v i s i o n}$ of Epidemiology, University of Utah, Salt Lake City, UT, United States \\ eDepartment of Medicine, University of Utah, Salt Lake City, UT, United States \\ fDepartment of Radiology, University of Utah, Salt Lake City, UT, United States \\ gDivision of General Surgery, Department of Surgery, University of Utah, Salt Lake City, UT, \\ United States
}

\section{Abstract}

Purpose-Studies suggest that "on-demand" radiography is equivalent to daily routine with regard to adverse events. In these studies, provider behavior is controlled. Pragmatic implementation has not been studied.

Materials and methods-This was a quasi-experimental, pre-post intervention study. Medical directors of two intervention ICUs requested pCXRs be ordered on an on-demand basis at one time point, without controlling or monitoring behavior or providing follow-up.

\footnotetext{
خrior Presentation: This work was presented in early form as an oral abstract at the 2016 Chest Annual Meeting in Los Angeles, CA. *Corresponding author at: Division of Cardiothoracic Surgery, Department of Surgery, University of Utah, Salt Lake City, UT, United States.

$1_{30}$ North 1900 East, 3C127, Salt Lake City, UT 84132.

230 North 1900 East, Salt Lake City, UT 84132.

330 North 1900 East, 3B110, Salt Lake City, UT 84132.

$\mathrm{KK}, \mathrm{RG}, \mathrm{RB}, \mathrm{JH}$, and YA additionally contributed to study design. AP, CZ, and MM additionally contributed to drafting of the manuscript.

Conflict of interest

None of the authors report any conflicts of interest related to this manuscript.

Other contributions

The authors are indebted to University of Utah Health who supported this effort.

Prior presentation

This work was presented in early form as an oral abstract at the 2016 Chest Annual Meeting in Los Angeles, CA.
} 
Results-A total of 11,994 patient days over 18 months were included. Combined characteristics: Age: 56.7, 66\% male, 96\% survival, APACHE II 14 (IQR: 11-19), mechanical ventilation (MV) (occurrences)/patient admission: mean 0.7 (SD: 0.6; range: 0-5), duration (hours) of MV: 21.7 (IQR: 9.8-81.4) and ICU LOS (days): 2.8 (IQR: 1.8-5.6). Average pCXR rate/patient/day before was 0.93 (95\% CI: 0.89-0.96), and 0.73 (95\% CI: 0.69-0.77) after. Controlling for severity, daily pCXR rate decreased by $21.7 \%(p<0.001)$, then increased by about $3 \% /$ month $(p=0.044)$. There was no change in APACHE II, mortality, and occurrences or duration of MV, unplanned re-intubations, ICU LOS.

Conclusions-In critically ill adults, pCXR reduction can be achieved in cardiothoracic and trauma/surgical patients with a pragmatic intervention, without adversely affecting patient care, outside a controlled study.

\section{Keywords}

Chest radiography (CXR); Portable radiography; Imaging; Daily; Routine

\section{Introduction}

Portable chest radiography (pCXR) is used frequently in intensive care units (ICUs), especially for postoperative cardiothoracic surgery and trauma patients. Extensive data demonstrates a low yield of clinical information when routine daily chest radiography is done for diverse ICU patient populations, including in postoperative surgical and cardiothoracic patients [1-7]. Studies have suggested that use of "on demand" chest radiography in the surgical ICU setting may increase the rate of positive findings found compared to "routine" chest radiography methodology [8,9]. Additionally, there are controlled clinical trials that demonstrate that moving from "routine" to "on-demand" chest radiography has no detrimental impact on patient outcome, though these trials have excluded cardiothoracic patients $[10,11]$. A recent meta-analysis found no difference among trials using restrictive ordering practices, but it was noted that studies did not rigorously assess for harm [12]. Smaller pre-post interventional studies have demonstrated no adverse effect on patient outcomes in the postoperative cardiothoracic and surgical populations when changing from "routine" to "on-demand" [13,14]. It is unknown if these same results can be achieved in routine clinical practice among any ICU population without at the same time mandating provider ordering behavior through the terms of a clinical trial or changes in order structure.

All previously observed studies of pCXR reduction in the ICU have managed provider behavior, through clinical trials, deliberate changes to ordering structure, and often by requiring a clinical indication for obtaining a radiograph [5,13-15]. To date, there are no studies that have evaluated the efficacy, durability or adverse events after a single time point intervention. Prior to undertaking this study, placement of a routine order for daily pCXR was standard culture in the cardiothoracic (CVICU) and surgical/trauma (SICU) ICUs at our institution. We chose to evaluate the impact of a pragmatic single time point intervention requesting on-demand testing as opposed to routine testing in our CVICU and SICU and to observe the efficacy and durability of the intervention and monitored patient outcomes for adverse events associated with this change. 
The clinical practice in two ICUs at our institution (CVICU and SICU) was to routinely obtain daily portable chest radiographs (pCXR) for patients with clinical conditions of the thorax (such as, pneumonia, chest trauma, respiratory failure), cardiothoracic post-operative patients and patients with thoracostomy tubes. Resident physicians and advanced practice clinicians (APCs) placed the order for a morning pCXR each prior evening in anticipation of morning rounds, despite a general recognition among the involved providers that this practice rarely resulted in meaningful patient changes. A clinical indication was required in placing the order. On-demand pCXRs were also routinely obtained after invasive procedures of the thorax (central venous catheters, thoracostomy tubes or postoperatively upon arrival to the ICU) or intubations. Subsequent on-demand pCXRs were obtained as clinically indicated rather than routinely ordered each day. The practice of obtaining routine daily pCXR was perpetuated by the practice culture in our ICUs where attending providers did not articulate that such ordering practice was not necessary and the perception by the ordering residents and APC providers that such daily pCXRs were expected by the attending staff and primary surgical teams.

\section{Materials and methods}

This was a prospective, pragmatic clinical practice intervention at a large tertiary academic medical center. Data collection was observational and approved by the Institutional Review Board as \#IRB_00084463 AM_00023786. The study design was a pre/post pragmatic clinical intervention in two intensive care units (ICUs): the cardiovascular ICU (CVICU) and the surgical ICU (SICU). The CVICU has an annual admission of approximately 700 patients who primarily comprise post-cardiac surgery patients, including heart and lung transplantation, as well as patients with durable and temporary mechanical circulatory support devices. A smaller percentage of CVICU patients included cardiology patients; these patients were not part of the intervention group and were excluded from data analysis. The SICU has an annual census of approximately 1000 to 1200 patients who comprise trauma, general and emergency surgery, abdominal organ transplantation, orthopedic, gynecologic and obstetric, vascular, otolaryngology and polytrauma patients. The control group was composed of two ICUs which did not receive the intervention [a medical ICU (MICU) and a neurocritical care ICU (NCCU)].

\subsection{Intervention}

The intervention was a single time point, pragmatic intervention in which the two medical directors of the CVICU and the SICU requested that pCXRs be ordered on an on-demand basis, rather than routinely each morning. The intervention was discussed and agreed upon at an in-person faculty meeting with the attending physicians from both intervention units ( $\sim 50 \%$ of the total 11 attendings present). This was followed by an electronic communication from the SICU medical director the same day, to the 11 attending physicians from both ICUs, detailing the request. The request included a graphic of the baseline rate of daily pCXR in the intervention units compared to the control units (left size of Fig. 1). The request did not include any language that could be perceived as incentive or threat, such as tracking behavior, financial remuneration or penalty, or follow-up. The APCs and residents who actually placed the pCXR orders were not directly involved in the intervention, though 
the decision to obtain a pCXR was made daily on multidisciplinary rounds, which included the surgeons and consultants. If a consultant wanted a pCXR, it was typically requested during multidisciplinary rounds. A second rounds occurred with the resident/APC and ICU attending in the evening to determine orders overnight and for morning rounds. ICU attendings were in house 27/7. The rationale for this was that the residents rotated on and off of the ICU service every 1-2 months, so in order to maximize the durability of a single time point intervention, only the attending physicians were included. As the ICU attendings were present on all rounds, they would be typically involved in all decisions to order pCXRs, including requests from consultants, and functioned as the "intervention." The rationale for this was that the residents rotated on and off of the ICU service every 1-2 months, so in order to maximize the durability of a single time point intervention, only the attending physicians were included.

\subsection{Data collection}

Observational data were collected from 9.8 months prior to the requested ordering change (7/1/14-4/24/15) through 8.9 months post (4/25/15-1/20/16) in the SICU and CVICU, and similarly in the control ICUs, NCCU and MICU. There was no washout period because we expected that the intervention would be enacted immediately. For the intervention group, patient and unit level data were extracted from the electronic health record (EHR) and manually extracted from the record. Variables included the following: patient demographics, pCXR use, illness severity (APACHE II scores), mortality, incidence and duration of mechanical ventilation [MV], ICU length of stay [LOS], reintubations, and case mix. Case mix was grouped under the following categorizations: group 1-cardiac or thoracic surgery; group 2-abdominal surgery, including abdominal transplant, abdominal aortic surgery or orthopedic surgery of the thoracoabdominal region, group 3-extremity surgery including vascular or orthopedic surgery, or head/neck surgery, and group 4-other surgical procedures. Control ICU pCXR/patient day data were qualitatively presented but not analyzed (Fig. 1).

\subsection{Analysis}

Data summaries were expressed as median (interquartile range, IQR) or frequency (percent). Patient characteristics were compared pre- and post-intervention using statistical tests including the Wilcoxon rank sum test for continuous variables and chi-squared, simulated chi-squared, or Fisher's exact test as appropriate for categorical variables. Interventionspecific changes were estimated using a poisson generalized estimating equation model implementing segmented regression (SR): Outcome ${ }_{t}=\beta_{0}+\beta_{1}$ Time $_{t}+\beta_{2}$ Intervention + $\beta_{3}$ Time-After-Intervention ${ }_{t}+\beta_{4 . . y} \mathbf{W}+e_{t}$ where $t$ indicates time in days, e is the error term, and $\mathbf{W}$ is a vector of patient severity/procedure variables. An auto-regressive order 1 covariance matrix was used to account for the correlation of daily pCXR data within a patient's visit. Coefficients were exponentiated for interpretation as ratio changes; and 95\% confidence intervals (CIs) and $p$-values were reported. Patient severity/procedure variables included: age, sex, ICU discharge condition, APACHE II, number of mechanical ventilation events, trach, reintubation within $48 \mathrm{~h}$, open chest, ECMO, VAD, ARDS, pneumonia, and ICU length of stay. The average pCXR rate pre- and post-intervention was calculated by the number of daily chest $\mathrm{X}$-rays divided by the total number of patient days in each period. The 
95\% CIs were estimated using the bootstrapping percentile method. Statistical significance was evaluated at $p<0.05$.

\section{Results}

There were 11,994 patient days from 1947 ICU admissions of 1729 patients (166 patients had more than one admission). Combined clinical characteristics are in Table 1, and briefly were: Age: 56.7(SD: 17.6), 66\% male, 96\% survival, APACHE II 14 (IQR: 11-19), MV (occurrences)/patient admission: mean 0.7 (SD: 0.6; range: 0-5), duration (hours) of MV: 21.7 (IQR: 9.8-81.4) and ICU LOS (days): 2.8 (IQR: 1.8-5.6). The average pCXR rate per patient per day before the intervention was 0.93 (95\% CI: 0.89-0.96), and after the date of the intervention it was 0.73 (95\% CI: 0.69-0.77). After controlling for patient severity characteristics, daily pCXR rate immediately following the intervention decreased by $21.7 \%$ $(p<0.001)$ (Table 3/Fig. 2). There was no change in APACHE II, mortality, and occurrences of MV, re-intubations, ICU LOS, or duration of MV (Table 2). The daily CXR rate following the intervention steadily increased over 8.8 months by about 3\%/month $(p=0.044)$ without further intervention (Table 3). There was no change in admission case mix (Table 2).

\section{Discussion}

In all fields of science, there is a well-recognized delay in the course of events from discovery to implementation and finally to adoption $[16,17]$. This interval of time can potentially include patient detriment. While multiple studies demonstrate the clinical benefit of transitioning from routine portable chest radiography in critically ill surgical and cardiovascular patients, all studies we found implemented change by controlling provider behavior or were monitored via clinical trials. Clinical trials are artificial relative to routine care, and while controlled changes to clinical ordering pathways can improve patient care, they also limit physician autonomy.

We sought to accelerate the implementation of a beneficial practice into routine care, and to furthermore test the efficacy of a single pragmatic clinical intervention to do this. Our study demonstrates that there can be a significant reduction of pCXRs in critically ill surgical and cardiovascular patients as a result of a single time point clinical intervention, despite it being outside of a clinical trial, and without forcing order placement or provider practice. This change occurred without adverse effects on patient care or evidence of harm, measured primarily through duration of MV, reintubations and ICU complications. Admission case mix was unchanged. It should be noted that there was a statistically significant decrease in the percentage of patients who discharged to the category ICU/IMCU from pre- to postimplementation. This was due to the closure of the IMCU unit just before the intervention period, affecting the disposition location. Most often this resulted in an increase in disposition to LTACs, and interestingly did not seem to increase duration of ICU LOS.

An important feature of our study was the observation that the durability of practice change is predictably limited. The clinical benefit, despite this limited durability, is appreciated by the graph (Fig. 1) showing the resultant decrease in PCXR during the intervention period. Given the intervention was a request by the medical director to the attending physicians, we 
expected and observed an initial drop in daily per patient pCXR rates. At this institution, during the study period, there were no methods in place to monitor provider ordering practices or compare testing across the attending physicians. Furthermore, the critical care attending physicians had 4 training background pathways, including general surgery, anesthesiology, emergency medicine, and cardiothoracic surgery. We observed that the lack of behavior tracking and group heterogeneity resulted in a general autonomy of practice of the individual attending physicians. Accordingly, we expected any decrease in pCXR use to be limited. Furthermore, the lack of order tracking, forcing orders, or regular reminders suggested to us that the attending group would quickly revert their practices to baseline after any change. Thus, it was surprising to us to observe the relative magnitude of the reduction in daily per patient $\mathrm{pCXR}$ rates and the duration of effect during the intervention period.

Simultaneously, our study highlights the importance of re-iterative process monitoring or quality assurance for durability of behavior change. We observed a steady increase in daily pCXR after the single time point intervention (Fig. 2). A single point intervention did influence ordering behavior and reduced pCXR utilization but did not result in a sustained change. To sustain such a change in practice, other ongoing measures or interventions are necessary. As described above, we intentionally did not provide follow-up reminders or requests to providers to encourage behavior change. We hypothesize that the effect of these reminders could potentially be significant-decreasing the steady return of pCXR rate/patient day towards baseline-and should be a focus of further study.

\section{Conclusions}

We found that a pragmatic single time point intervention to reduce radiography resulted in a significant reduction in daily pCXRs without changes in re-intubation, LOS, or duration of MV. The intervention implemented here could serve as a model for other ICUs wishing to implement evidence based changes without forcing order pathways or monitoring provider behavior.

\section{Acknowledgments}

JT had full access to all the data in the study, takes responsibility for the integrity of the data, the accuracy of the data analysis, and the integrity of the submission as a whole, from inception to published article, including study design, drafting of the manuscript and final approval. All authors (JT, KK, AP, CZ, MM, RG, RB, JH, YA) contributed substantially to the study through acquisition of data, analysis and interpretation, revised the manuscript critically for important intellectual content, provided final approval, and agreed to be accountable for all aspects of the work.

\section{Role of the sponsors}

This study was supported, in part, by the University of Utah Study Design and Biostatistics Center, with funding in part from the National Center for Research Resources and the National Center for Advancing Translational Sciences, National Institutes of Health, through Grant 5UL1TR001067-02 (formerly 8UL1TR000105 and UL1RR025764).

\section{Abbreviations \\ CXR chest radiograph \\ pCXR portable chest radiograph}




$\begin{array}{ll}\text { ICU } & \text { intensive care unit } \\ \text { CVICU } & \text { cardiovascular intensive care unit } \\ \text { SICU } & \text { surgical intensive care unit } \\ \text { NCCU } & \text { neurocritical care unit } \\ \text { MICU } & \text { medical intensive care unit } \\ \text { IMCU } & \text { intermediate care unit } \\ \text { LTAC } & \text { long term acute care } \\ \text { SNF } & \text { skilled nursing facility }\end{array}$

APACHE II Acute Physiology and Chronic Health Evaluation II

ECMO extracorporeal membrane oxygenation

VAD ventricular assist device

MODS multiple organ dysfunction syndrome

PE pulmonary embolism

VAP ventilator associated pneumonia

MV mechanical ventilation

IQR inter-quartile range

CI confidence interval

\section{References}

1. Silverstein DS, Livingston DH, Elcavage J, et al. The utility of routine daily chest radiography in the surgical intensive care unit. J Trauma. 1993; 35(4):643-6. [PubMed: 8411291]

2. Cruz J, Ferra M, Kasarabada A, et al. Evaluation of the clinical utility of routine daily chest radiography in intensive care unit patients with tracheostomy tubes: a retrospective review. $\mathrm{J}$ Intensive Care Med. 2014:1-5.

3. Bhagwanjee S, Muckart DJJ. Routine daily chest radiography is not indicated for ventilated patients in a surgical ICU. Intensive Care Med. 1996; 22(12):1335-8. [PubMed: 8986482]

4. Fong Y, Whalen GF, Hariri RJ, et al. Utility of routine chest radiographs in the surgical intensive care unit. A prospective study. Arch Surg. 1995; 130(7):764-8. [PubMed: 7611867]

5. Graat ME, Kroner A, Spronk PE, et al. Elimination of daily routine chest radiographs in a mixed medical-surgical intensive care unit. Intensive Care Med. 2007; 33(4):639-44. [PubMed: 17333118]

6. Graham RJ, Meziane MA, Rice TW, et al. Postoperative portable chest radiographs: optimum use in thoracic surgery. J Thorac Cardiov Sur. 1998; 115(1):45-50.

7. Tolsma M, Bentala M, Rosseel PMJ, et al. The value of routine chest radiographs after minimally invasive cardiac surgery: an observational cohort study. J Cardiothorac Surg. 2014; 9

8. Resnick S, Inaba K, Karamanos E, et al. Clinical relevance of the routine daily chest X-ray in the surgical intensive care unit. Am J Surg. 2016 
9. Reeb J, Falcoz P-E, Olland A, et al. Are daily routine chest radiographs necessary after pulmonary surgery in adult patients? Table 1. Interact Cardiovasc Thorac Surg. 2013; 17(6):995-8. [PubMed: 23956264]

10. Clec'h C, Simon P, Hamdi A, et al. Are daily routine chest radiographs useful in critically ill, mechanically ventilated patients? A randomized study. Intensive Care Med. 2007; 34(2):264-70. [PubMed: 17994222]

11. Hejblum G, Chalumeau-Lemoine L, Ioos V, et al. Comparison of routine and on-demand prescription of chest radiographs in mechanically ventilated adults: a multicentre, clusterrandomised, two-period crossover study. Lancet. 2009; 374(9702):1687-93. [PubMed: 19896184]

12. Ganapathy A, Adhikari NK, Spiegelman J, et al. Routine chest X-rays in intensive care units: a systematic review and meta-analysis. Crit Care. 2012; 16(2):R68. [PubMed: 22541022]

13. Salehi M, Saberi K, Rahmanian M, et al. Assessment of limited chest X-ray technique in postcardiac surgery management. Ann Card Anaesth. 2017; 20(1):38-41. [PubMed: 28074793]

14. Mets O, Spronk PE, Binnekade J, et al. Elimination of daily routine chest radiographs does not change on-demand radiography practice in post-cardiothoracic surgery patients. J Thorac Cardiovasc Surg. 2007; 134(1):139-44. [PubMed: 17599499]

15. Hendrikse KA, Gratama JW, Hove W, et al. Low value of routine chest radiographs in a mixed medical-surgical ICU. Chest. 2007; 132(3):823-8. [PubMed: 17873192]

16. Balas EA, Boren SA. Managing clinical knowledge for health care improvement. Yearb Med Inform. 2000; 1:65-70.

17. Morris ZS, Wooding S, Grant J. The answer is 17 years, what is the question: understanding time lags in translational research. J R Soc Med. 2011; 104(12):510-20. [PubMed: 22179294] 


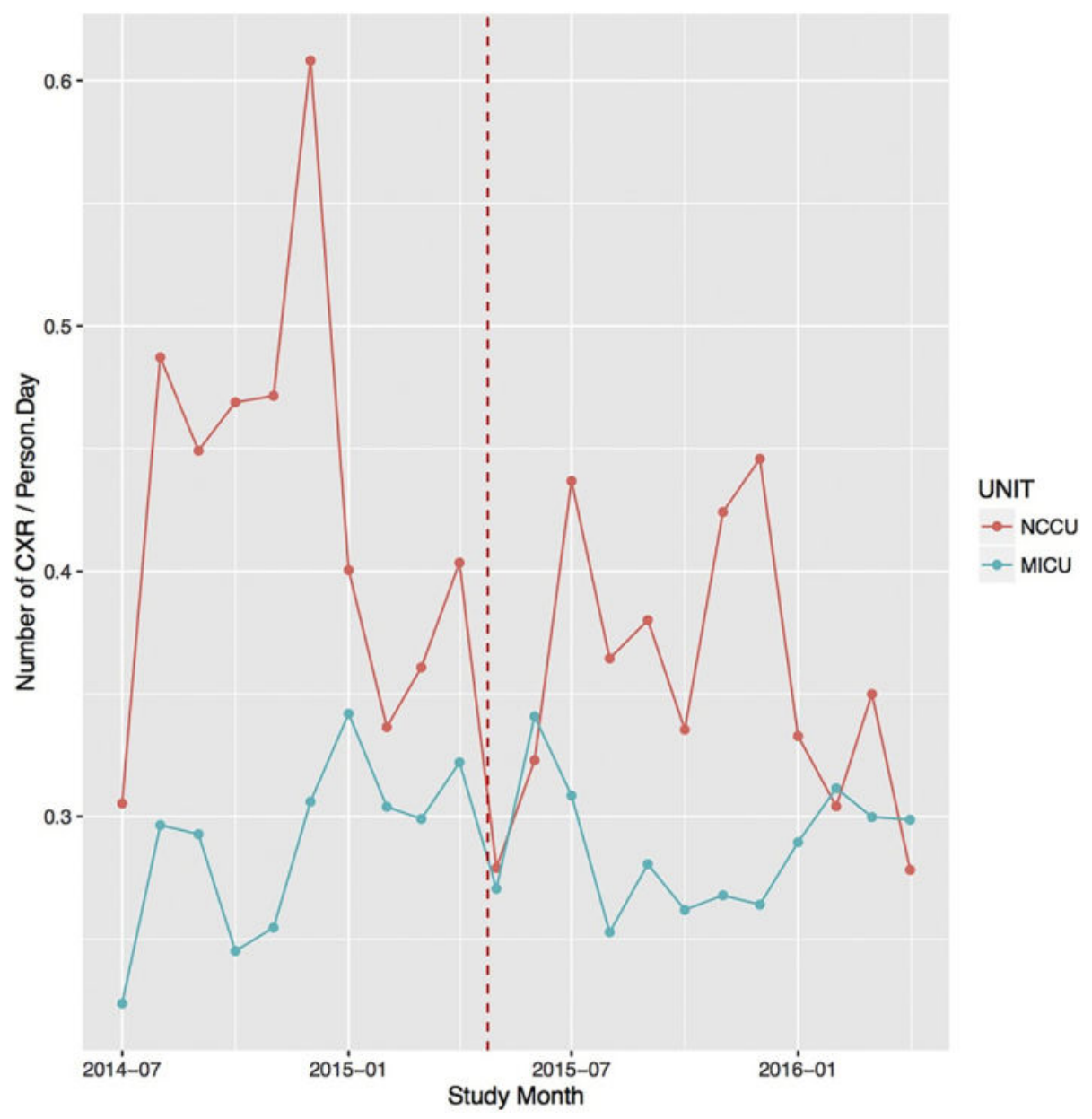

Fig. 1.

Control ICU rate of $\mathrm{pCXR/patient/day.} \mathrm{Control} \mathrm{ICU} \mathrm{rate} \mathrm{of} \mathrm{pCXR} /$ patient/day was stable before and after the intervention. (Figure displays monthly data for clarity). 


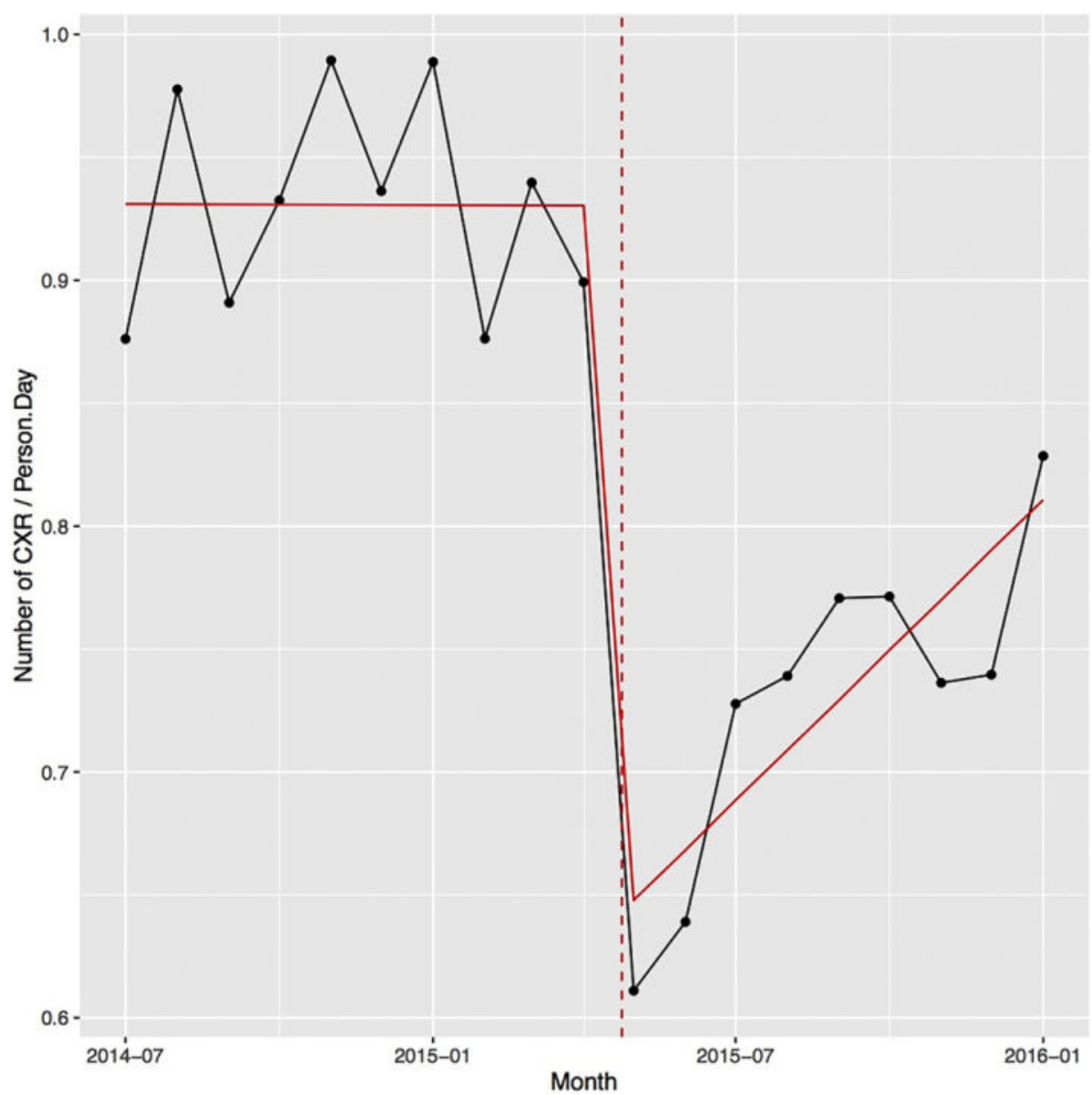

Fig. 2.

Intervention ICU rate of $\mathrm{pCXR/patient/day.} \mathrm{Intervention} \mathrm{ICU} \mathrm{rate} \mathrm{of} \mathrm{pCXR} / \mathrm{patient} / \mathrm{day}$ dropped after intervention, rising slowly without further intervention. (Figure displays monthly data for clarity). 


\section{Table 1}

Descriptive Summary at the ICU admission level $(N=1947)$.

\begin{tabular}{|c|c|}
\hline Variable & Summary \\
\hline Number of ICU Admissions (Pre) & $1011(52 \%)$ \\
\hline Number of ICU Admissions (Post) & $936(48 \%)$ \\
\hline pCXR/patient/day & $0.8(0.3,1)$ \\
\hline Age & $59(45,69.5)$ \\
\hline Male & $1279(66 \%)$ \\
\hline \multicolumn{2}{|l|}{ Unit location } \\
\hline Cardiovascular ICU & $885(50 \%)$ \\
\hline Surgical ICU & $873(50 \%)$ \\
\hline ICU Mortality & $79(4 \%)$ \\
\hline ICU Length of Stay & $2.8(1.8,5.6)$ \\
\hline \multicolumn{2}{|l|}{ ICU discharge location } \\
\hline Floor & $1520(78 \%)$ \\
\hline Home/Other & $122(6 \%)$ \\
\hline ICU/IMCU & $172(9 \%)$ \\
\hline LTAC/SNF & $50(3 \%)$ \\
\hline Other & $2(0 \%)$ \\
\hline APACHE II & $14(11,19)$ \\
\hline \multicolumn{2}{|l|}{ Intubations per patient } \\
\hline 0 & $652(33 \%)$ \\
\hline 1 & $1183(61 \%)$ \\
\hline $2+$ & $112(6 \%)$ \\
\hline Tracheostomy & $103(5 \%)$ \\
\hline Total Ventilator Hours & $21.7(9.8,81.4)$ \\
\hline \multicolumn{2}{|l|}{ Reintubations within $48 \mathrm{~h}$} \\
\hline 0 & $1886(97 \%)$ \\
\hline 1 & $49(3 \%)$ \\
\hline 2 & $3(0 \%)$ \\
\hline \multicolumn{2}{|l|}{ Additional characteristics } \\
\hline Open chest & $47(2 \%)$ \\
\hline ECMO & $45(2 \%)$ \\
\hline Ventricular assist device & $92(5 \%)$ \\
\hline ARDS & $35(2 \%)$ \\
\hline Hemo/pneumothorax & $6(0 \%)$ \\
\hline MODS & $25(1 \%)$ \\
\hline PE & $4(0 \%)$ \\
\hline VAP & $34(2 \%)$ \\
\hline Pneumonia & $80(4 \%)$ \\
\hline Aspiration & $22(1 \%)$ \\
\hline
\end{tabular}

J Crit Care. Author manuscript; available in PMC 2019 April 01. 


\begin{tabular}{ll}
\hline Variable & Summary \\
\hline 1 & $752(39 \%)$ \\
2 & $442(23 \%)$ \\
3 & $245(13 \%)$ \\
4 & $508(26 \%)$ \\
\hline
\end{tabular}




\section{Table 2}

Descriptive summary at the admission level by group.

\begin{tabular}{|c|c|c|c|}
\hline Variable & $\operatorname{Pre}(N=1011)$ & Post $(N=936)$ & P-value \\
\hline Age & $59(44,69)$ & $59(45.8,70)$ & 0.87 \\
\hline \multicolumn{4}{|l|}{ Gender } \\
\hline Female & $349(35 \%)$ & $319(34 \%)$ & 0.84 \\
\hline Male & $662(65 \%)$ & $617(66 \%)$ & - \\
\hline \multicolumn{4}{|l|}{ Unit location } \\
\hline CVICU & $445(49 \%)$ & $440(52 \%)$ & 0.21 \\
\hline SICU & $465(51 \%)$ & $408(48 \%)$ & - \\
\hline ICU Mortality & $40(4 \%)$ & $39(4 \%)$ & 0.91 \\
\hline ICU length of stay (days) & $2.8(1.8,5.6)$ & $2.8(1.8,5.5)$ & 0.26 \\
\hline \multicolumn{4}{|l|}{ ICU discharge location } \\
\hline Floor & $731(72 \%)$ & $789(84 \%)$ & $<0.001$ \\
\hline HOME/OTHER & $60(6 \%)$ & $62(7 \%)$ & - \\
\hline ICU/IMCU & $158(16 \%)$ & $14(1 \%)$ & - \\
\hline LTAC/SNF & $20(2 \%)$ & $30(3 \%)$ & - \\
\hline Other & $1(0 \%)$ & $1(0 \%)$ & - \\
\hline APACHE II & $15(11,19)$ & $14(11,19)$ & 0.52 \\
\hline \multicolumn{4}{|l|}{ Intubations per patient } \\
\hline 0 & $343(34 \%)$ & $309(33 \%)$ & 0.79 \\
\hline 1 & $613(61 \%)$ & $570(61 \%)$ & - \\
\hline $2+$ & $55(5 \%)$ & $57(6 \%)$ & - \\
\hline Tracheostomy & $58(6 \%)$ & $45(5 \%)$ & 0.36 \\
\hline Mechanical ventilation (hours) & $21.1(9.9,82.6)$ & $22.3(9.8,79.9)$ & 0.83 \\
\hline \multicolumn{4}{|l|}{ Reintubations within 48 h (\#) } \\
\hline 0 & $988(98 \%)$ & $898(97 \%)$ & 0.11 \\
\hline 1 & $19(2 \%)$ & $30(3 \%)$ & - \\
\hline 2 & $1(0 \%)$ & $2(0 \%)$ & - \\
\hline \multicolumn{4}{|l|}{ Additional characteristics } \\
\hline Open chest & $26(3 \%)$ & $21(2 \%)$ & 0.64 \\
\hline ECMO & $23(2 \%)$ & $22(2 \%)$ & 0.91 \\
\hline VAD & $45(4 \%)$ & $47(5 \%)$ & 0.55 \\
\hline ARDS & $14(1 \%)$ & $21(2 \%)$ & 0.15 \\
\hline Hemo/Pneumothorax & $2(0 \%)$ & $4(0 \%)$ & 0.44 \\
\hline MODS & $13(1 \%)$ & $12(1 \%)$ & 0.99 \\
\hline $\mathrm{PE}$ & $3(0 \%)$ & $1(0 \%)$ & 0.63 \\
\hline VAP & $19(2 \%)$ & $15(2 \%)$ & 0.64 \\
\hline Pneumonia & $36(4 \%)$ & $44(5 \%)$ & 0.21 \\
\hline Aspiration & $9(1 \%)$ & $13(1 \%)$ & 0.30 \\
\hline \multicolumn{4}{|l|}{ Patient admission case mix group } \\
\hline 1 & $380(38 \%)$ & $372(40 \%)$ & 0.77 \\
\hline
\end{tabular}

J Crit Care. Author manuscript; available in PMC 2019 April 01. 


\begin{tabular}{clll}
\hline Variable & Pre $(\boldsymbol{N}=\mathbf{1 0 1 1})$ & Post $(\boldsymbol{N}=\mathbf{9 3 6})$ & P-value \\
\hline 2 & $237(23 \%)$ & $205(22 \%)$ & - \\
3 & $128(13 \%)$ & $117(12 \%)$ & - \\
4 & $266(26 \%)$ & $242(26 \%)$ & - \\
\hline
\end{tabular}




\section{Table 3}

Estimates of intervention effect using a Poisson GEE model.

\begin{tabular}{|c|c|c|c|c|}
\hline Effects & Unadjusted \% change & p-Value & Adjusted \% change ${ }^{a}$ & p-Value \\
\hline Immediate change in daily pCXR rate after intervention & $-23.2 \%(-32 \%,-13.2 \%)$ & $<0.001$ & $-24.7 \%(-33.3 \%,-15 \%)$ & $<0.001$ \\
\hline Change per month in daily pCXR rate since study began & $-0.2 \%(-1.5 \%, 1.2 \%)$ & 0.79 & $-0.7 \%(-2 \%, 0.6 \%)$ & 0.26 \\
\hline $\begin{array}{l}\text { Additional change per month in daily } \mathrm{pCXR} \text { rate post } \\
\text { intervention }\end{array}$ & $1.2 \%(-1.1 \%, 3.6 \%)$ & 0.30 & $2.8 \%(0.7 \%, 4.9 \%)$ & 0.008 \\
\hline
\end{tabular}

${ }^{a}$ Adjusting for patient severity characteristics. 